\title{
Chapter 1 \\ Introduction: Overview of Our Research on Impacts of the Fukushima Dai-ichi Nuclear Power Plant Accident on Fish and Fishing Grounds
}

\author{
Kaoru Nakata and Hiroya Sugisaki
}

\begin{abstract}
As a result of the Fukushima Dai-Ichi Nuclear Power Plant accident in March 2011, a large volume of radionuclides was released into the environment, thus contaminating marine and freshwater systems. The Fisheries Research Agency has conducted research beginning soon after the accident. Our research addressed the contamination processes of radionuclides (mainly radiocesium) through water, sediments, and food chains, in both marine and freshwater systems, based on a large volume of original in situ data. Our research has also provided important information on when and how marine fish have been contaminated. This chapter gives an overview of our research.
\end{abstract}

Keywords Fukushima - Radionuclides - Radiocesium - Marine and freshwater systems $\bullet$ Contamination process

\subsection{Objectives of Our Research}

As a result of the Great East Japan Earthquake on March 11, 2011, and the resulting tsunami, all power supplies to the No. 1 through No. 4 nuclear reactors at Tokyo Electric Power's Fukushima I Nuclear Power Plant (FNPP) were lost because of submergence and electrical discharge. As a result, core meltdowns occurred in the No. 1 to No. 3 reactors and hydrogen explosions sequentially occurred in No. 1, No. 3, and No. 4 nuclear reactors. By March 15, a large amount of radioactive materials had been released into the environment as fallout, which contaminated both marine

\footnotetext{
K. Nakata $(\bowtie)$

Fisheries Research Agency, Queen's Tower B 15F, 2-3-3 Minato Mirai,

Nishi-ku, Yokohama, Kanagawa 220-6115, Japan

e-mail: may31@affrc.go.jp

H. Sugisaki

National Research Institute of Fisheries Sciences, Fisheries Research Agency,

2-12-4, Fukuura, Yokohama, Kanagawa 236-8648, Japan
} 
and freshwater systems. On April 2, heavily contaminated water was found at an intake of the No. 2 reactor. In addition, several leakages of contaminated water around FNPP, as well as a release of low-level contaminated water by Tokyo Electric Power Company (TEPCO), had occurred by May 2011.

The Fisheries Research Agency (FRA) has conducted research to clarify the impacts of the FNPP accident on fish and fishing grounds since soon after the accident. Our research has mainly involved monitoring radionuclides in fish and their habitats and conducting studies to clarify the dynamics of radionuclides in both marine and freshwater systems. This book aims to introduce the results of our research, the bulk of which was conducted from 2011 to 2013. An overview of our results is given next.

\subsection{Seawater and Plankton (Part I)}

FNPP-derived radiocesium has accumulated in marine food webs via seawater intake and predator-prey interactions. Information on the spatiotemporal distribution of radiocesium concentration in seawater and plankters is therefore important for comprehending the accumulation and dynamics of radiocesium in pelagic ecosystems. Although a large amount of contaminated water was released into the ocean, by 2012 the ${ }^{137} \mathrm{Cs}$ concentrations in the surface seawater around Japan (Fig. 1.1), except for the waters off the Pacific coast of Tohoku district (Fig. 1.1), had dropped to levels seen before the accident (Table 1.1). The concentrations off the Pacific coast of Tohoku district in 2012 were still about ten times higher than those before the accident, but they had decreased to the level seen before the accident by late 2013 (FRA 2014).

Chapter 2 summarizes the dispersion process of FNPP-derived radioactive cesium (Cs) in seawater, based on observatory data and numerical simulation. A considerable amount of radioactive Cs from FNPP dispersed from the western North Pacific eastward to the central North Pacific during the first year after the accident. It then dispersed not only eastward but also northward and southward in the central North Pacific in the subsequent second and third years. Research by the FRA also shows transportation of the contaminated water into the subtropical zone beneath the Kuroshio Extension.

Chapter 3 shows the temporal variability of ${ }^{137} \mathrm{Cs}$ concentrations in zooplankters off the Joban coast and Sendai Bay, on the Pacific coast of the middle to southern Tohoku district. ${ }^{137} \mathrm{Cs}$ concentrations in both seawater and zooplankton have decreased during our research period since June 2011. However, the rate decrease in seawater was faster than in zooplankton, which resulted in a high apparent concentration ratio (aCR) for zooplankton (Chap. 3). We also show that the aCR value measured in zooplankton accurately describes the progress of ${ }^{137} \mathrm{Cs}$ contamination in zooplankton, from the beginning of the FNPP accident (dynamic nonequilibrium state) to the restoration phase (dynamic equilibrium state). 


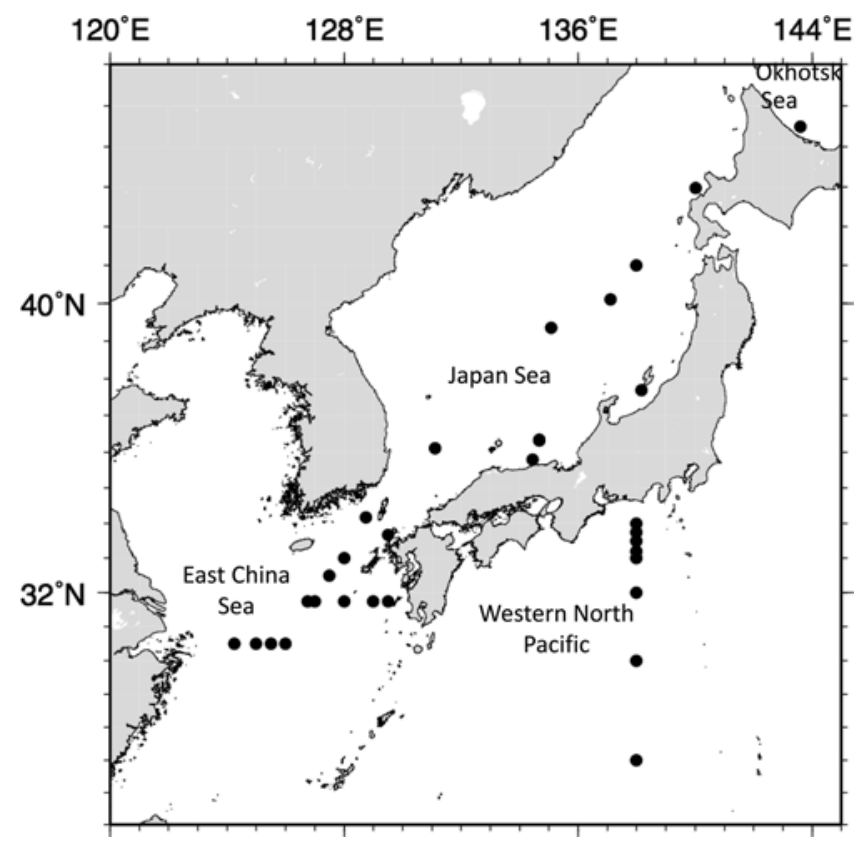

Fig. 1.1 Sampling points of surface water for analyzing the radiocesium concentrations around Japan

Table 1.1 Comparisons of ${ }^{137} \mathrm{Cs}$ concentration in surface waters around Japan between 2012 and periods before the accident at Fukushima Dai-ichi Nuclear Power Plant in March 2011

\begin{tabular}{l|l|l|l}
\hline \multirow{2}{*}{ Sea area } & \multirow{2}{*}{} & \multicolumn{2}{l}{137 Cs concentration $(\mathrm{mBq} / \mathrm{kg})$} \\
\cline { 3 - 4 } Sea of Okhotsk & Month surveyed (2012) & 2012 & $2001-2010$ \\
\hline Sea of Japan & Jun & $1.9 \pm 0.37$ & $2.2-\mathrm{ND}$ \\
\hline East China Sea & Jun-Nov & $2.4-2.0$ & $2.9-\mathrm{ND}$ \\
\hline (Kuroshio) & Jun-Oct & $2.0-1.4$ & $2.4-1.4$ \\
\hline
\end{tabular}

Source: http://www.fra.affrc.go.jp/eq/Nuclear_accident_effects/H24seika.pdf (in Japanese)

- Chapter $2 .{ }^{134} \mathrm{Cs}$ and ${ }^{137} \mathrm{Cs}$ in the Seawater Around Japan and in the North Pacific (H. Kaeriyama)

- Chapter 3. Temporal Changes in ${ }^{137} \mathrm{Cs}$ Concentration in Zooplankton and Seawater off the Joban-Sanriku Coast, and in Sendai Bay, After the Fukushima Dai-ichi Nuclear Accident (H. Kaeriyama) 


\subsection{Sediments and Benthos (Part II)}

Intensive monitoring of radiocesium in marine organisms by the Fukushima Prefectural Fisheries Experimental Station showed that the radiocesium concentration in demersal fish was higher, and showed slower decline, compared with pelagic fish (Wada et al. 2013). This phenomenon might have been largely influenced by the distribution and dynamics of radiocesium in sediment. The FRA has investigated the spatial and temporal distribution of radiocesium concentrations in marine sediment, its translation from contaminated sediment to benthos, and the probable food of demersal fish. The results are summarized in Part II.

Chapter 4 shows the spatial distribution of the radiocesium concentration in the top $14 \mathrm{~cm}$ of sea sediment off the coast of northern Ibaraki to Fukushima, with 5-min horizontal resolution. There was a high concentration band along 100-m isobaths, where the concentration of ${ }^{137} \mathrm{Cs}$ reached a maximum of $1,240 \mathrm{~Bq} / \mathrm{kg}$-dry. When assessing radiocesium transportation from sea sediments to a marine demersal ecosystem, information is required not only on the concentration but also on the biological ingestibility of sea sediment radiocesium. To assess radiocesium transportation from sea sediments to a benthic ecosystem, radiocesium concentration in the organic fraction of sea sediments $\left(\mathrm{Cs}_{\text {org }}\right)$ was analyzed (Chap. 5), and showed horizontal distribution of $\mathrm{Cs}_{\text {org }}$ off the coast of northern Ibaraki to Fukushima and Sendai Bay. $\mathrm{Cs}_{\text {org }}$ of sea sediments was significantly higher than that of bulk sediments $\left(\mathrm{Cs}_{\text {bulk }}\right)$. We suggest that $\mathrm{Cs}_{\text {org }}$ can be used as an indicator of the potential effects of sediment radiocesium on the demersal ecosystem.

The FRA also monitored the behavior of particulate matter, which is closely related to that of sediment radiocesium, at a depth of $32 \mathrm{~m}$ off Iwaki, Fukushima, by automated observatory systems (Chap. 6). The behavior was largely influenced by waves, and particulate matter was resuspended and transported with water movement during high waves. We show that the combination of waves and currents resulting from meteorological disturbance is one of the important processes in the transport of suspended particle material off the Fukushima coast.

On the basis of the results from field investigations and rearing experiments using a benthic polychaete (Perinereis aibuhitensis) with highly contaminated sediment collected at the station $1 \mathrm{~km}$ off FNPP, the FRA estimated the transport of radiocesium from contaminated sediment to benthic organisms off the coast of Fukushima (Chap. 7). These results suggest that the intake of radiocesium through the benthic food web is limited for benthic organisms, even if the sediments are highly contaminated.

- Chapter 4. Three-Dimensional Distribution of Radiocesium in Sea Sediment derived from the Fukushima Dai-ichi Nuclear Power Plant (D. Ambe et al.)

- Chapter 5. Radiocesium Concentrations in the Organic Fraction of Sea Sediments (T. Ono et al.)

- Chapter 6. Bottom Turbidity, Boundary Layer Dynamics, and Associated Transport of Suspended Particulate Materials off the Fukushima Coast (H. Yagi et al.) 
- Chapter 7. Investigation of Radiocesium Translation from Contaminated Sediment to Benthic Organisms (Y. Shigenobu et al.)

\subsection{Marine Fish (Part III)}

Off the Fukushima coast, the percentage of fish with a radiocesium concentration higher than $100 \mathrm{~Bq} / \mathrm{kg}$-wet (the standard value of radiocesium in foods) accounted for more than $90 \%$ of the fish caught off Fukushima in April 2011. The percentage had declined to $0.6 \%$ by October 2014, according to the Fukushima Prefectural Fisheries Experimental Station (2014). The concentration trend was different between pelagic and demersal fish. Part III describes the characteristics of the temporal variations of the concentration and their background mechanisms for small epipelagic fish (sardine and anchovy) and demersal fish (cod and flatfish).

In addition to the intrusion of the contaminated waters from FNPP, some radionuclides were delivered to marine fish and their habitat through the atmospheric pathway. Chapter 8 describes evidence of impacts via the atmospheric pathway on small epipelagic fish off the coast of southern Ibaraki and Chiba Prefectures, before the direct release of contaminated water into the ocean. After the release, fluctuations in the radiocesium concentration in fish muscles were synchronized with the decreasing concentration in the seawater near the fishing ground; the radiocesium concentration in fish muscles reached a maximum of $31 \mathrm{~Bq} / \mathrm{kg}$-wet in July 2011, after which it declined gradually (Chap. 9).

Decline of the radiocesium concentration in demersal fish seemed to be slower compared with pelagic fish, but this varied individually and across species. It is still unclear which species or individuals of demersal fish showed high radiocesium concentrations. Chapter 10 shows differences in radiocesium concentration in the Pacific cod (Gadus microcephalus) across year-classes and also suggests that the difference could be explained by ontogenetic changes in diet and seasonal changes in vertical distribution.

Chapter 11 describes three features of the contamination histories of the Japanese flounder (Paralichthys olivaceus) after the accident by analyzing the observed spatiotemporal changes in Cs concentration, a comparison of the dynamics of Cs concentration across year-classes, and simulation studies: (1) high Cs values with high variation in the first year after the accident, (2) low Cs values with their minimum values peaking around autumn 2011, and (3) lower Cs values observed for 2011 year-class and younger than 2010 year-class and older. A hypothesis on the background mechanisms is also discussed.

- Chapter 8. Detection of ${ }^{131} \mathrm{I},{ }^{134} \mathrm{Cs}$, and ${ }^{137} \mathrm{Cs}$ Released into the Atmosphere from FNPP in Small Epipelagic Fishes, Japanese Sardine, and Japanese Anchovy, off the Kanto Area, Japan (T. Morita et al.)

- Chapter 9. Radiocesium Concentration of Small Epipelagic Fishes (Sardine and Japanese Anchovy) off the Kashima-Boso Area (K. Takagi et al.) 
- Chapter 10. Why Do the Radionuclide Concentrations of Pacific Cod Depend on Body Size? (Y. Narimatsu et al.)

- Chapter 11. Radiocesium Contamination Histories of Japanese Flounder (Paralichthys olivaceus) after the 2011 Fukushima Nuclear Power Plant Accident (Y. Kurita et al.)

\subsection{Mechanisms of Severe Contamination in Fish (Part IV)}

Decreasing trends of radiocesium have generally been found for pelagic fish since summer 2011 and for demersal fish since winter 2012 (http://www.fra.affrc.go.jp/ eq/Nuclear_accident_effects/H24seika.pdf). However, extremely contaminated fat greenlings (Hexagrammos otakii), with $25,800 \mathrm{~Bq} / \mathrm{kg}$-wet of radiocesium, which is the highest value except for fish collected in the FNPP port (Chap. 14), were caught off Ota River within a 20-km radius of FNPP. The FRA investigated the causes and mechanisms for the occurrence of the extremely contaminated fat greenlings.

Based on the radiocesium concentrations in 236 greenlings that had been collected off the coast of Fukushima after the accident, the probability of the occurrence of extremely contaminated fat greenlings was calculated assuming a normal distribution (Chap. 12). The probability was exceedingly low, at less than $2.794 \times 10^{-6}$, yet the concentration found was almost equivalent to that frequently observed for the greenlings caught in the FNPP port.

The contamination process for the extremely contaminated fat greenlings was also investigated by analyses of beta-ray emission from otoliths and using a biokinetic model; the results are shown in Chap. 13. Analyses of the beta-ray emission from the otoliths showed that the fat greenlings were in the highly contaminated environment in the period just after the FNPP accident. Simulation of the ${ }^{137} \mathrm{Cs}$ concentration in fat greenlings using the biokinetic model showed that the fat greenlings had their origin in the FNPP port just after the accident.

Contamination levels of fish caught in the FNPP port are summarized in Chap. 14, and radiocesium concentrations in fat greenlings, Japanese rockfish, and spotbelly rockfish are shown to be higher than in other species. Relationships among beta rays emitted from otoliths, ${ }^{90} \mathrm{Sr}$, and radiocesium in the whole body without internal organs were confirmed for Japanese rockfish.

- Chapter 12. Evaluating the Probability of Catching Fat Greenlings (Hexagrammos otakii) Highly Contaminated with Radiocesium off the Coast of Fukushima (Y. Shigenobu et al.)

- Chapter 13. Analysis of the Contamination Process of the Extremely Contaminated Fat Greenling by Fukushima-Derived Radioactive Material (T. Watanabe et al.)

- Chapter 14. Contamination Levels of Radioactive Cesium in Fat Greenling Caught at the Main Port of the Fukushima Dai-ichi Nuclear Power Plant (K. Fujimoto et al.) 


\subsection{Freshwater Systems (Part V)}

Contamination of freshwater systems by the FNPP accident was primarily caused by fallout from the FNPP. Part V describes factors affecting variation among freshwater systems, fish species, and individuals.

Contamination levels were compared across Lake Hayama, Lake Akimoto, and Lake Tagokura in Fukushima Prefecture (Chap. 15). Radiocesium concentrations of the lake water, bottom sediment, plankton, and fish were significantly correlated with the surface soil radiocesium content near the lake sites. In Lake Hayama, with the highest contamination level of the three lakes, factors affecting the radiocesium concentration level in several fish species were considered (Chap. 16). Body size and feeding habit seemed to influence the variation among fish species in Lake Hayama.

The radiocesium concentration level in ayu (Plecoglossus altivelis) was analyzed in five rivers in Fukushima Prefecture between summer 2011 and autumn 2013 (Chap. 17). The concentrations of radiocesium in ayu were shown to have decreased during the study period. Our research also shows a positive correlation between the concentrations of radiocesium in the internal organs and the muscle of ayu $(r=0.746$, $p=0.006$ ). However, the median concentration in the muscle was $14.5 \%$ that of the median concentration in the internal organs, which shows that a small proportion (about $15 \%$ ) of the ingested food from the riverbed appears to be transferred to the muscle.

The contamination levels in salmonid fish were also investigated in Lake Chuzenji, central Honshu Island, Japan, in Tochigi Prefecture (160 km from the station) (Chap. 18). In Lake Chuzenji, substantial accumulations of radiocesium were confirmed in the muscle of hatchery-reared kokanee (Oncorhynchus nerka) and masu salmon (Oncorhynchus masou). Rearing experiments controlling for water and food radiocesium levels revealed that radiocesium contamination of fish is an ongoing process, and that radiocesium is accumulated in fish via the food chain (Chap. 19).

- Chapter 15. Comparison of the Radioactive Cesium Contamination Level of Fish and their Habitat Among Three Lakes in Fukushima Prefecture, Japan, After the Fukushima Fallout (K. Matsuda et al.)

- Chapter 16. Radiocesium Concentrations and Body Size of Freshwater Fish in Lake Hayama 1 Year After the Fukushima Dai-ichi Nuclear Power Plant Accident (K. Takagi et al.)

- Chapter 17. Spatiotemporal Monitoring of ${ }^{134} \mathrm{Cs}$ and ${ }^{137} \mathrm{Cs}$ in Ayu, Plecoglossus altivelis, a Microalgae-Grazing Fish, and in Their Freshwater Habitats in Fukushima (J. Tsuboi et al.)

- Chapter 18. Radiocesium Concentrations in the Muscle and Eggs of Salmonids from Lake Chuzenji, Japan, After the Fukushima Fallout (S. Yamamoto et al.)

- Chapter 19. Assessment of Radiocesium Accumulation by Hatchery-Reared Salmonids After the Fukushima Nuclear Accident (S. Yamamoto et al.) 
Open Access This chapter is distributed under the terms of the Creative Commons Attribution Noncommercial License, which permits any noncommercial use, distribution, and reproduction in any medium, provided the original author(s) and source are credited.

\section{References}

Fukushima Prefectural Fisheries Experimental Station (2014) http://www.pref.fukushima.lg.jp/ uploaded/attachment/930000.pdf. Accessed 27 Dec 2014. [in Japanese]

FRA (2014) http://www.fra.affrc.go.jp/eq/Nuclear_accident_effects/H25seika.pdf. Accessed 20 Nov 2014. [in Japanese]

Wada T, Nemoto Y, Shimamura S, Fujita T, Mizuno T, Sohtome T, Kamiyama K, Morita T, Igarashi S (2013) Effects of the nuclear disaster on marine products in Fukushima. J Environ Radioact 124:246-254 